\title{
Efficacy of Rituximab Monotherapy for an Elderly Hemodialysis Patient with Primary Cardiac Lymphoma
}

\author{
Yasuyoshi Morita ${ }^{1}$, Mitsuhiro Matsuda ${ }^{2}$, Terufumi Yamaguchi ${ }^{1}$, Mika Sakaguchi ${ }^{3}$, \\ Shinya Rai ${ }^{1}$, Yoshitaka Kanai ${ }^{1}$, Chikara Hirase ${ }^{1}$, Kazunobu Kawanishi ${ }^{1}$, Junichi Miyatake ${ }^{1}$, \\ Takahiro Shimada ${ }^{1}$, Yoichi Tatsumi ${ }^{1}$, Takashi Ashida ${ }^{1}$, \\ Yasuhiro Maeda ${ }^{1}$ and Akihisa Kanamaru ${ }^{1}$
}

\begin{abstract}
We report a case of primary cardiac lymphoma (PCL) occurring in a 76-year-old man during maintenance hemodialysis. Chest computed tomography (CT) revealed a tumor with pericardial effusion in the left ventricular posterior wall. Cytological examination of the pericardial fluid revealed monotonous lymphoid cells positive for B-cell markers, and clonal immunoglobulin heavy chain gene rearrangement was detected, indicating B-cell lymphoma. Rituximab monotherapy was administered biweekly at the therapeutic level on hemodialysis. The follow-up chest CT showed tumor disappearance with pericardial fluid after two courses of therapy. Rituximab monotherapy was effective for an elderly hemodialysis patient with PCL.
\end{abstract}

Key words: primary cardiac lymphoma, chronic renal failure, rituximab monotherapy, serum rituximab level

(Inter Med 49: 2163-2166, 2010)

(DOI: 10.2169/internalmedicine.49.4018)

\section{Introduction}

Primary cardiac lymphoma (PCL) is a rare extranodal non-Hodgkin's lymphoma (NHL) exclusively located in the heart and/or pericardium with no evidence of extracardiac dissemination (1). This disease accounts for only $1.3 \%$ of cardiac tumors and $0.5 \%$ of extranodal lymphomas (2). Histopathologic diagnosis is important as it influences treatment and prognosis; however, a definitive diagnosis is not always easy to make from biopsy specimens (3). In some cases, open chest biopsy is difficult to perform especially for older patients. On the other hand, rituximab, a chimeric monoclonal antibody that binds to CD20 antigen, is highly active and well tolerated as a first-line single-agent therapy for indolent NHL $(4,5)$. Moreover, rituximab monotherapy administered every two weeks has been proven to be highly effective for an older patient with intravascular lymphoma after chemotherapy (6). Here, we report a rare case of PCL in an elderly patient with chronic renal failure $(\mathrm{CRF})$ on hemodialysis who was successfully treated with rituximab monotherapy.

\section{Case Report}

A 76-year-old man was referred to our hospital in February 2006 because of a mass and fluid retention in the pericardium. The patient was receiving hemodialysis three times a week having developed CRF 5 years previously. While on hemodialysis in the previous hospital, he developed sudden dyspnea due to fluid retention in the pericardium which was detected on ultrasonic cardiography. During pericardial drainage at the previous hospital, he developed no serious cardiovascular or respiratory symptoms. He was transferred to our hospital with the following clinical data: blood pressure, 120/72 $\mathrm{mmHg}$; heart rate, 78 beats/min; oxygen saturation, $98 \%$ in room air. He had a performance status (PS) of 3 and was in wheelchair. Chest computed tomography (CT) revealed a mass $(6.6 \times 5.4 \mathrm{~cm})$ in the pericardium over the left ventricle, with no enlargement of lymph nodes around

${ }^{1}$ Department of Hematology, Kinki University School of Medicine, Osaka-Sayama, ${ }^{2}$ Department of Internal Medicine, PL General Hospital, Tondabayashi and ${ }^{3}$ Department of Nephrology, Kinki University Sakai Hospital, Sakai

Received for publication May 29, 2010; Accepted for publication July 9, 2010

Correspondence to Dr. Yasuyoshi Morita, morita@int3.med.kindai.ac.jp 
(A)

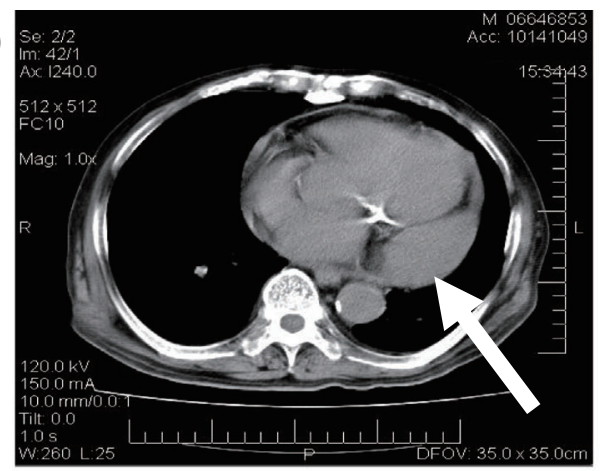

(B)

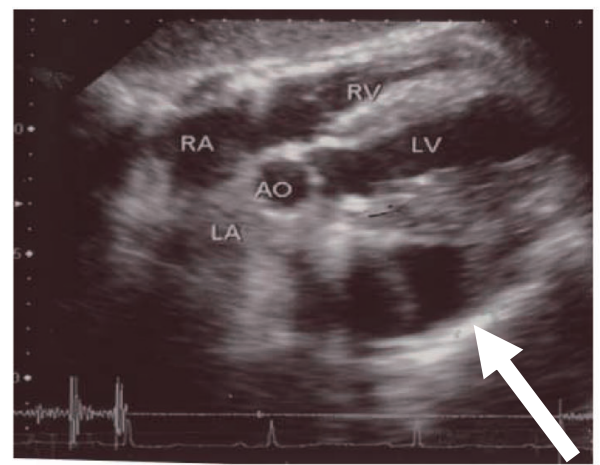

(C)

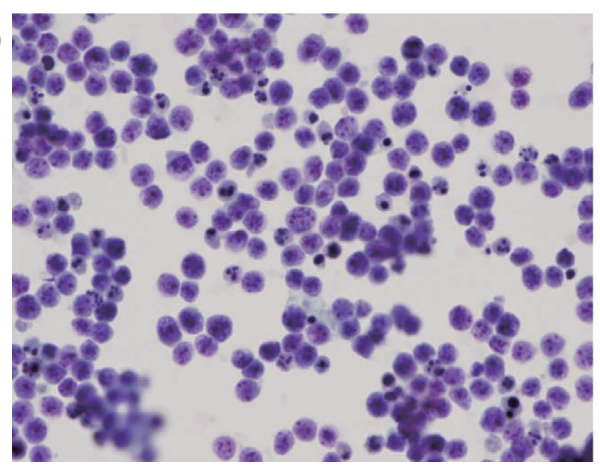

(D)

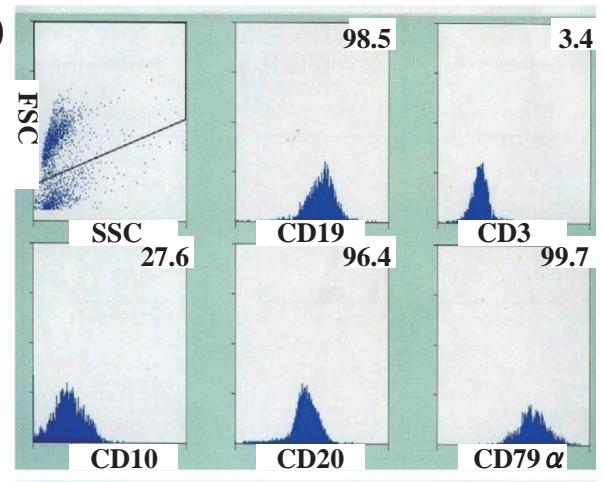

(E)

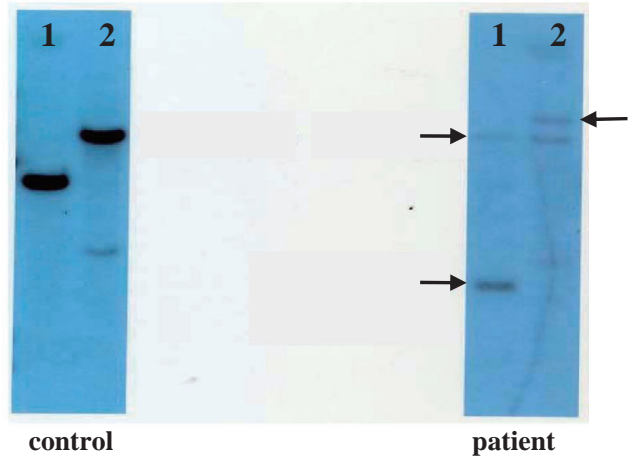

(F)

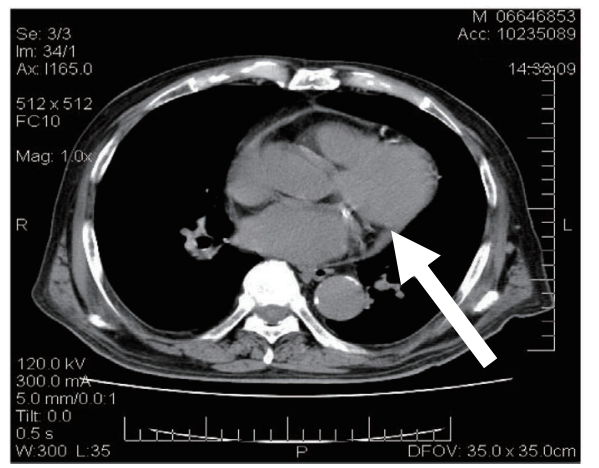

Figure 1. Diagnostic imaging of primary cardiac lymphoma (PCL). (A) Chest computed tomography (CT) revealed a large mass (arrow) occupying the left ventricular posterior wall (LVPW) before rituximab monotherapy. (B) Transthoracic echocardiography detected an echo free space (arrow), which showed pericardial effusion. (C) Cytological analysis of pericardial fluid revealed an increased number of large lymphoid cells with irregular nuclei (Wright-Giemsa staining, $\times 400$ ). (D) Expression of CD19, CD79 $\alpha, \mathrm{CD10}, \mathrm{CD20}$, and $\mathrm{CD} 3$ in pericardial fluid as demonstrated by flow cytometric analysis. The number of upper-right corner indicates \% positive rate for isotopic antibody. CD: cluster differentiation, FSC: forward scatter, SSC: side scatter. (E) Immunoglobulin heavy chain (IgH) gene rearrangements in pericardial fluid as shown by Southern blotting analysis. The cells were digested with BamHI+Hind III (1) or Hind III (2). The arrows indicate IgH gene rearrangements. (F) Chest CT demonstrated disappearance of the mass (arrow) occupying the LVPW following rituximab monotherapy. RA: right atrium, RV: right ventricle, LA: left atrium, LV: left ventricle, Ao: aorta.

the aorta or trachea or axillary lymph nodes (Fig. 1A). Neck to pelvis CT also revealed no lesions, including nodal lesions, other than the pericardial lesion, suggesting a primary cardiac tumor. Transthoracic echocardiography showed an echo free space in the left ventricular posterior wall (LVPW) suggestive of pericardial effusion (Fig. 1B). Following pericardiocentesis in our hospital, we attempted to confirm a diagnosis of malignant lymphoma based on indirect evidence through morphologic evaluation, use of cell surface markers, and monoclonality of the cytologic specimen. This proce- dure was adopted because tissue biopsy of the mass in the LVPW was technically difficult and performing thoracotomy in this elderly patient seemed too risky. Cytologic analysis of pericardial fluid revealed increased number of large lymphoid cells with irregular nuclei (Fig. 1C). These cells were positive for CD10, CD19, CD20, CD79 $\alpha$, and negative for CD3 as shown by flow cytometric analysis (Fig. 1D). Moreover, clonal immunoglobulin heavy chain gene rearrangement was detected, indicating B-cell lymphoma (Fig. 1E). Cytogenetic analysis of lymphoma cells from pericardial 
Table 1. Serum Rituximab Levels on Hemodialysis

\begin{tabular}{c|c|c|}
\multirow{2}{*}{ Time (h) } & \multicolumn{2}{|c|}{ Rituximab levels $(\mu \mathrm{g} / \mathrm{mL})$} \\
\cline { 2 - 3 } 0 & Patient & Control patient \\
\hline 2 & 0 & 0 \\
\hline 4 & 78.9 & 30.9 \\
\hline 6 & 289.3 & 168.8 \\
\hline 12 & 324.0 & 207.9 \\
\hline 24 & 216.2 & 150.1 \\
\hline 48 & 201.3 & 145.8 \\
\hline
\end{tabular}

The rituximab level during hemodialysis in the present patient was compared with that in a control diffuse large B-cell lymphoma (DLBCL) patient with normal renal function. In both patients, rituximab was administered at the same time on the same day, and blood samples were collected at the same time points. Hemodialysis was performed for $\mathbf{4}$ hours on the first day. The start time of hemodialysis was 1 hour after rituximab administration.

fluid using the G-banding method indicated a normal karyotype. Physical examination revealed the patient to be anemic without surface lymphadenopathy and hepatosplenomegaly. Laboratory tests showed a hemoglobin concentration of 11.8 $\mathrm{g} / \mathrm{dL}$ and a serum creatinine level of $8.4 \mathrm{mg} / \mathrm{dL}$, indicating CRF. Other laboratory data revealed a serum C-reactive protein (CRP) level of $3.0 \mathrm{mg} / \mathrm{dL}$, a soluble interleukin 2 receptor (sIL-2R) concentration of $2,638 \mathrm{U} / \mathrm{mL}$ and lactase dehydrogenase (LDH) level within the normal range. The antibody for the human immunodeficiency virus was negative. Bone marrow aspiration and biopsy showed no involvement of lymphoma cells. These findings led to a diagnosis of CD20-positive B-cell lymphoma (Ann Arbor classification; stage IE) (7). The patient was informed of the need for combination chemotherapy containing rituximab. However, because of old age and poor PS, and he could barely tolerate maintenance hemodialysis three times a week, it would be stressful for him to frequently visit the hospital to receive supportive therapy for myelosuppression after chemotherapy. Furthermore, it would be difficult to determine the optimal dose of chemotherapy due to his CRF. For these reasons, the patient elected to receive rituximab monotherapy. Jillella et al reported that rituximab represents an unproblematic, safe and successful treatment option for patients with end-stage renal failure on hemodialysis (8). To prove that rituximab was not removed by the dialysis solution and that it was maintained at a therapeutic level in serum, we measured the blood concentration of rituximab by enzyme-linked immunosorbent assay using FALCO Biosystems, Inc. (Kyoto, Japan) $(8,9)$. Four-hour hemodialysis was started one hour after rituximab administration. As a control, we measured rituximab level in another patient who was newly diagnosed with diffuse large B-cell lymphoma (DLBCL) with a normal renal function (creatinine clearance, $94.2 \mathrm{~mL} / \mathrm{min}$ ) after obtaining consent (Table 1). Rituximab was administered dur- ing hemodialysis. The vital signs of the patient were stable during hemodialysis, with no sign of infusion-related reaction to rituximab. Blood concentrations at each time point were higher in the patient with CRF than in the patient with normal renal function, suggesting the maintenance of an effective therapeutic level in a renal failure patient (Table 1). The follow-up chest CT showed tumor disappearance with pericardial fluid after two courses of rituximab monotherapy every two weeks (Fig. 1F). During the total six courses of rituximab monotherapy, the patient was maintained in complete remission. However, further therapy could not be carried out because the patient did not consent to the next therapy. Serological data following rituximab monotherapy indicated that the serum levels of CRP, sIL-2R and LDH were within the normal range. However, the patient experienced a relapse of the mass with pericardial effusion and pleural effusion 10 months after diagnosis, and died 14 months after diagnosis due to progressive disease.

\section{Discussion}

Ceresoli et al reported that cardiac metastasis of malignant lymphoma, as well as a majority of cases of PCL, commonly occurs on the right side of the heart (10). Although the localization of the cardiac tumor was not determined histologically, the present case was a very rare case of PCL localized on the left side of the heart as determined by imaging modalities. PCL is classified mainly as DLBCL (60\%-81\% of cases) and less frequently as immunoblastic, Burkitt type, or lymphoblastic lymphoma. In more than $90 \%$ of cases, lymphoma cells belong to the B-cell lineage (11). In the present case, since cardiac tumor biopsy via thoracotomy seemed risky in this elderly patient with concomitant morbidity, biopsy was not performed, and the diagnosis of B-cell lymphoma was made by examination of morphology, cell surface markers, and monoclonality of the cytologic specimen (12). In Japan, only 2 case reports of PCL in the pericardium with no lesions in the myocardium have been reported to date (13).

PCL is considered to have a poor prognosis with a median survival time of 7 months (14). Due to the limited number of cases, no standard therapy has yet been established. However, it has been suggested that PCL should be treated similar to other bulky aggressive NHLs (10). Therefore, we recommended rituximab-containing combination chemotherapy with an anthracycline as the key drug (THPCOP therapy) for the patient. However, the patient refused this combination chemotherapy. Nakagawa et al showed that rituximab monotherapy was safe and effective for primary cardiac B-cell lymphoma (15). In the present case, the patient received rituximab monotherapy at $375 \mathrm{mg} / \mathrm{m}^{2}$ biweekly on hemodialysis. The blood level of rituximab during hemodialysis was maintained at a higher level than that in a DLBCL patient with normal renal function, with the peak level reached 6 hours after administration, followed by a similar pharmacokinetic pattern as that in the control pa- 
tient. Jillella et al (8) measured the blood level of rituximab before and after each hemodialysis course and showed that rituximab was not removed by hemodialysis. Our data not only support their finding but also provide very valuable information including detailed pharmacokinetic measurements from the start to 48 hours after administration.

The standard treatment for PCL has not yet been established. This case has provided valuable data demonstrating that the mean survival time can be prolonged with rituximab monotherapy. Also, rituximab monotherapy is safe and effective for patients with primary cardiac B-cell lymphoma who are unable to receive chemotherapy because of advanced age and maintenance hemodialysis.

\section{Acknowledgement}

This work was supported in part by The Osaka Medical Research Foundation for Incurable Disease. We would like to thank Satoko Nagayama and Sayuri Yoshida for their excellent technical assistance.

\section{References}

1. Burke A, Virmani R. Tumors of the heart and great vessels. Series III. In: Atlas of Tumor Pathology. Fascicle 16. Rosai J, Sobin LH, Eds. Armed Forces Institute of Pathology, Washington, DC, 1996: 171-179.

2. McAllister HA Jr, Fenoglio JJ Jr. Tumors of the cardiovascular system. Series II. In: Atlas of Tumor Pathology. Fascicle 15. Armed Forces Institute of Pathology, Washington, DC, 1979: 99100.

3. Chim CS, Chan AC, Kwong YL, Liang R. Primary cardiac lymphoma. Am J Hematol 54: 79-83, 1997.

4. Hainsworth JD, Litchy S, Burris HA 3rd, et al. Rituxmab as firstline and maintenance therapy for patients with indolent nonHodgkin's lymphoma. J Clin Oncol 20: 4261-4267, 2002.
5. Colombat P, Salles G, Brousse N, et al. Rituximab (anti-CD20 monoclonal antibody) as single first-line therapy for patients with follicular lymphoma with a low tumor burden: clinical and molecular evaluation. Blood 97: 101-106, 2000.

6. Davis TS. Intravascular lymphoma presenting with cauda equina syndrome: treated with CHOP and rituxan. Leuk Lymphoma 44 : 887-888, 2003.

7. Carbone PP, Kaplan HS, Musshoff K, Smithers DW, Tubiana M. Report of the committee Hodgkin's disease staging classification. Cancer Res 31: 1860-1861, 1971.

8. Jillella AP, Danier PM, Kallab AM, Ustun C. Treatment of a patient with end-stage renal disease with rituximab: Pharmacokinetic evaluation suggests rituximab is not eliminated by hemodialysis. Am J Hematol 71: 219-222, 2002.

9. Iacona I, Lazzarino M, Avanzini MA, et al. Rituximab (IDECC2B8): validation of a sensitive enzyme-linked immunoassay applied to clinical pharmacokinetic study. Ther Drug Monit 22: 295$301,2000$.

10. Ceresoli GL, Ferreri AJ, Bucci E, Ripa C, Ponzoni M, Villa E. Primary cardiac lymphoma in immunocompetent patients: diagnostic and therapeutic management. Cancer 80: 1497-1506, 1997.

11. Chalabreysse L, Berger F, Lorie R, Devouassoux G, Cordier JF, Thivolet-Bejui F. Primary cardiac lymphoma in immunocompetent patients: a report of three cases and review of literature. Virchows Arch 441: 456-461, 2002.

12. Nonami A, Takenaka K, Kamezaki K, et al. Successful treatment of primary cardiac lymphoma by rituximab-CHOP and high-dose chemotherapy with autologous peripheral blood stem cell transplantation. Int J Hematol 85: 264-266, 2007.

13. Nagamine K, Noda $H$. Two cases of primary cardiac lymphoma presenting with pericardial effusion and cardiac tamponade. Jpn Circ J 54: 1158-1164, 1990.

14. Rolla G, Bertero MT, Pastena G, et al. Primary lymphoma of the heart: a case report and review of literature. Leuk Res 26: 117120, 2002.

15. Nakagawa Y, Ikeda U, Hirose M, et al. Successful treatment of primary cardiac lymphoma with monoclonal CD20 antibody (Rituximab). Circ J 68: 172-173, 2004.

(C) 2010 The Japanese Society of Internal Medicine

http://www.naika.or.jp/imindex.html 